\title{
Design and Analysis of a Linear Type Electromagnetic Stirrer
}

\author{
S. Milind, V. Ramanarayanan \\ Power Electronics Group, Department of Electrical Engineering \\ Indian Institute of Science \\ Bangalore-560012, INDIA \\ Email: milind@ee.iisc.ernet.in, vram@ee.iisc.ernet.in
}

\begin{abstract}
Electromagnetic stirring (EMS) of metallic alloys is mainly used to refine the grain structure of castings. This technique results in increased homogeneity of the cast alloys. In this paper a design oriented approach to a Linear Electromagnetic Stirrer is presented. A mathematical model of such a stirrer is proposed to obtain electromagnetic field solutions. The field solutions so obtained are compared with those obtained from the finite-element model. The influence of excitation frequency and current on the induced current density and axial force and their variation with radius have been investigated. Experimental results obtained from a prototype are presented.
\end{abstract}

\section{INTRODUCTION}

The process of semi-solid forming(SSF) is becoming popular for the manufacture of several components in automotive industries. SSF combines the high mechanical strength feature of forging together with low cost performance feature of casting. The process utilizes the thixotropic nature of the slurry having globular structure instead of dendritic structure of the solidifying mass. The non-dendritic feedstock is primarily produced by mechanical or electromagnetic stirring of the metal during freezing. Electromagnetic stirring has an edge over mechanical stirring in that no physical contact exists between the stirrer and the metal. Also, stirring is uniform throughout the metal.

The two types of stirrers of stirrers commonly used in practice are the linear stirrer and the rotary stirrer [1]- [3]. A linear stirrer uses travelling field whereas, the rotary stirrer uses rotating magnetic field to produce swirling action in the metal pool.

This paper focuses on linear stirrer from the design point of view. The design problem in such a stirrer is the evaluation of electromagnetic fields for a given geometry, electrical excitation, and frequency of excitation of the stirrer, and relate the same to the induced eddy currents, mechanical forces and power transferred to the metal pool. The electromagnetic problem is solved with simplifying assumptions to obtain closed form field solutions.

\section{PRINCIPLE OF WORKING}

Fig. 1 shows the structure of an electromagnetic stirrer. The working principle is similar to that of a 2 pole linear induction motor. The stirrer is excited by three phase currents through space displaced three phase windings A, B and C. The three phase currents in the space displaced coils setup

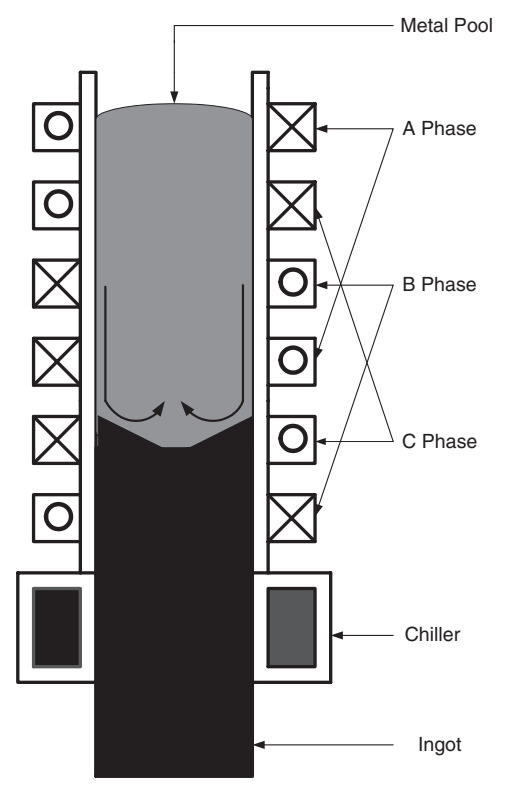

Fig. 1. Structure of a Linear Electromagnetic Stirrer

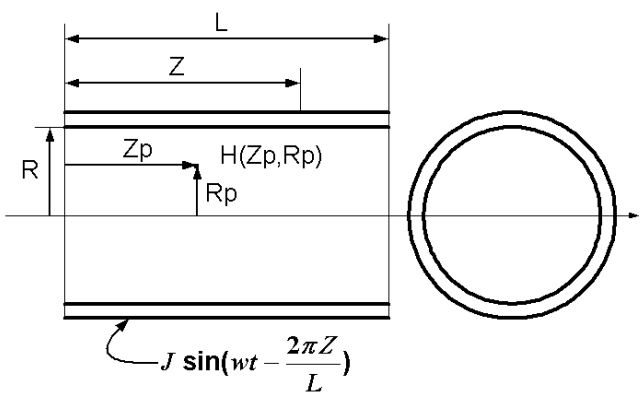

Fig. 2. Three phase Excitation approximated to a linear current sheet

travelling magnetic field in the metal pool with linear velocity of $L \cdot f \mathrm{~m} / \mathrm{sec}$, where $L$ is the length of field coil and $f$ is the frequency of excitation. If the end effects are neglected (when $L>>R$ ), the three phase coils may be considered equivalent to an excitation with a linear current density of as shown in Fig. 2 [4], [5]. Such an approximation is possible by obtaining the three lumped currents in the individual coils by their space harmonics and superimposing the three currents into an equivalent travelling wave with a linear current density 


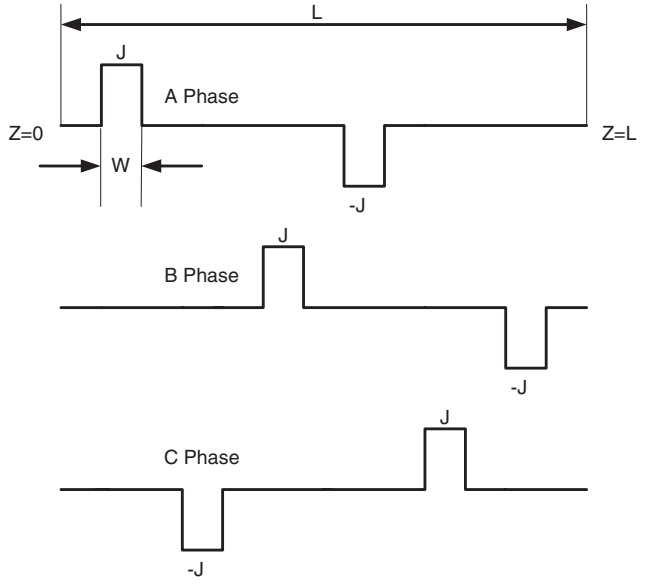

Fig. 3. Distributed current density in the windings

as shown in Fig. 2 and 3. The resulting axial field is almost constant; the radial field is proportional to the radius and leads the axial field by 90 . The end effects may be consolidated in a suitable correction factor. The analysis is carried out for a stirrer with $\mathrm{R}=0.04 \mathrm{~m}$ and $\mathrm{L}=0.16 \mathrm{~m}$. The correction factor $\left(C_{r}\right)$ on account of end effect is found to be 0.42 . Since the field is varying with time, emf is induced in the single turn metal core, which in turn establishes eddy currents in the molten metal. The eddy current and the magnetic field interact to give radial and axial mechanical forces. It is seen that the radial forces average to zero whereas the axial forces average in the direction of phase sequence resulting in movement of the metal.

\section{Mathematical Model}

\section{A. Field in the air gap}

Let $d H_{r}\left(Z_{p}, R_{p}, Z\right)$ and $d H_{z}\left(Z_{p}, R_{p}, Z\right)$ respectively be the radial and axial component of field at point $\left(Z_{p}, R_{p}\right)$ on account of the current in the elemental ring having $\mathrm{N}$ turns carrying a current density of $\mathrm{J} A$ located at $\mathrm{Z}$. Then,

$$
\begin{aligned}
& d H_{r}\left(Z_{p}, R_{p}, Z\right)= \\
& \quad \frac{N R J}{4 \pi} \int_{0}^{2 \pi} \frac{\left(Z-Z_{p}\right) \cos \phi d \phi}{\left[R_{p}^{2}+R^{2}-2 R R_{p} \cos \phi+\left(Z_{p}-Z\right)^{2}\right]^{\frac{3}{2}}} d Z \\
& d H_{z}\left(Z_{p}, R_{p}, Z\right)= \\
& \quad \frac{N R J}{4 \pi} \int_{0}^{2 \pi} \frac{\left(R-R_{p} \cos \phi\right) d \phi}{\left[R_{p}^{2}+R^{2}-2 R R_{p} \cos \phi+\left(Z_{p}-Z\right)^{2}\right]^{\frac{3}{2}}} d Z
\end{aligned}
$$

Let $H_{r a}\left(Z_{p}, R_{p}\right)$ and $H_{z a}\left(Z_{p}, R_{p}\right)$ respectively be the radial and axial component of field at point $\left(Z_{p}, R_{p}\right)$ due to current density of $\pm \mathrm{J} \mathrm{A} / \mathrm{m}$ in the A-phase coils of $\mathrm{N}$ turns having width $W$. Then,

$$
\begin{aligned}
& H_{r a}\left(Z_{p}, R_{p}\right)= \\
& \quad \int_{0}^{W} d H_{r a}\left(Z_{p}, R_{p}, Z\right) d Z-\int_{0}^{W} d H_{r a^{\prime}}\left(Z_{p}, R_{p}, Z\right) d Z
\end{aligned}
$$

$$
\begin{aligned}
& H_{z a}\left(Z_{p}, R_{p}\right)= \\
& \quad \int_{0}^{W} d H_{z a}\left(Z_{p}, R_{p}, Z\right) d Z-\int_{0}^{W} d H_{z a^{\prime}}\left(Z_{p}, R_{p}, Z\right) d Z
\end{aligned}
$$

Accordingly $H_{r b}\left(Z_{p}, R_{p}\right), H_{r c}\left(Z_{p}, R_{p}\right), H_{z b}\left(Z_{p}, R_{p}\right)$ and $H_{z c}\left(Z_{p}, R_{p}\right)$ are defined.

The axial and radial field at any point $\left(Z_{p}, R_{p}\right)$ as a function of time when the coils are excited by three phase currents is given by

$$
\begin{aligned}
& H_{z}\left(Z_{p}, R_{p}, t\right)=H_{z a}\left(Z_{p}, R_{p}\right) \cdot \sin (\omega t)+ \\
& H_{z b}\left(Z_{p}, R_{p}\right) \cdot \sin \left(\omega t-\frac{2 \pi}{3}\right)+H_{z c}\left(Z_{p}, R_{p}\right) \cdot \sin \left(\omega t-\frac{4 \pi}{3}\right) \\
& H_{r}\left(Z_{p}, R_{p}, t\right)=H_{r a}\left(Z_{p}, R_{p}\right) \cdot \sin (\omega t)+ \\
& H_{r b}\left(Z_{p}, R_{p}\right) \cdot \sin \left(\omega t-\frac{2 \pi}{3}\right)+H_{r c}\left(Z_{p}, R_{p}\right) \cdot \sin \left(\omega t-\frac{4 \pi}{3}\right)
\end{aligned}
$$

Fig. 4 and 5 show the $\operatorname{axial}\left(H_{z}\right)$ and $\operatorname{radial}\left(H_{r}\right)$ field for different radius and at different instants of time for $\mathrm{L}=0.16 \mathrm{~m}$, $\mathrm{R}=0.042 \mathrm{~m}, \mathrm{f}=50 \mathrm{~Hz}, \mathrm{~N}=10$, width of the $\operatorname{coil}(\mathrm{W})=8 \mathrm{~mm}$ and excitation current $=200 \mathrm{~A}(\mathrm{rms})$. It is seen that the axial field is nearly constant along the radius whereas the radial field can be approximated as proportional to the radius. Also, the axial field lags the radial field by $90^{\circ}$.

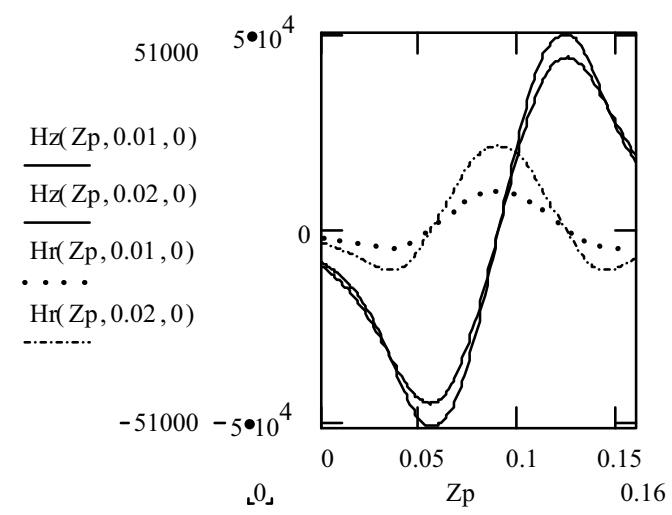

Fig. 4. Axial and Radial field at $r=0.01$ and $0.02 \mathrm{~m}$ and $\mathrm{t}=0 \mathrm{sec}$

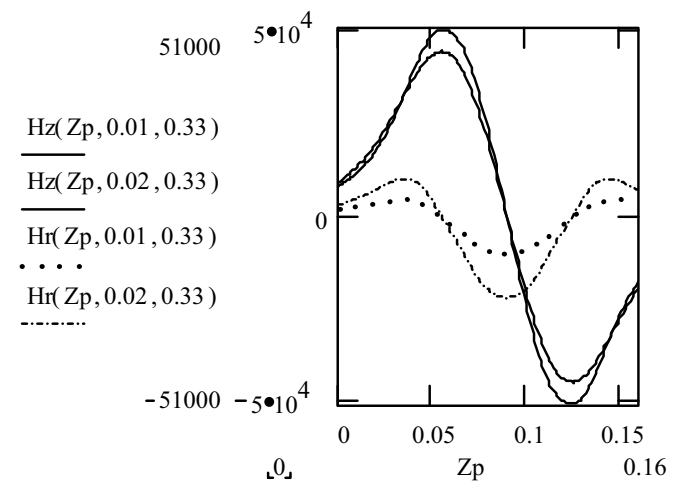

Fig. 5. Axial and Radial field at $r=0.01$ and $0.02 \mathrm{~m}$ and $\mathrm{t}=0.33 \mathrm{sec}$ 


\section{B. Estimation of correction factor}

From Fig. 3 fundamental (space) phase component of $\mathbf{J}$ is given by

$$
J_{1}=\frac{4}{\pi} J \sin \left(\frac{\pi}{18}\right)
$$

Equivalent single phase fundamental

$$
J_{1}^{\prime}=1.5 \frac{4}{\pi} J \sin \left(\frac{\pi}{18}\right)
$$

Ideally (when $L / R>>1$ ) this value is equal to the peak value of the $\mathrm{H}$ field $\left(H_{0}\right)$ i.e. field is completely in the axial direction with no radial component. With L/R being finite (in our case 4) the end effect can be consolidated in a suitable correction factor. If $C_{r}$ is the correction factor then,

$$
\begin{gathered}
C_{r} \cdot J_{1}^{\prime}=50000 \\
C_{r}=0.42
\end{gathered}
$$

Thus we conclude that in the idealized case the $\mathrm{H}$ field has only axial component travelling with a velocity of $L \cdot f \mathrm{~m} / \mathrm{sec}$ and is governed by the equation $H_{z}=H_{0} \sin \left(\omega t-\frac{2 \pi Z}{L}\right)$.

\section{Field intensities in the metal pool}

Since the field is varying with time, voltage is induced in the metal pool. This emf establishes eddy currents in the molten metal. Fig. 6 shows the formulation of the field problem with the field entirely in the $\mathrm{Z}$ direction, the induced voltage and the eddy current in the $\phi$ direction [6]. Let

$\mathrm{H}=$ Magnetic field intensity at radius $\mathrm{r}(\mathrm{A} / \mathrm{m})$

$v_{\phi}=$ Induced emf in circle of radius $\mathrm{r}$ in $\phi$ direction $(\mathrm{V})$

$J_{\phi}=$ Eddy current density at radius $\mathrm{r}$ in $\phi$ direction $\left(\mathrm{A} / \mathrm{m}^{2}\right)$

$\sigma=$ Conductivity of metal $\left(\mathrm{Sm}^{-1}\right)$

$\mu=$ Permeability of the metal $(\mathrm{H} / \mathrm{m})$

The following are the governing equations.

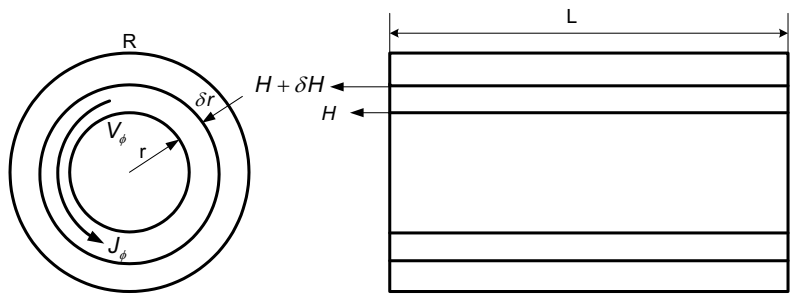

Fig. 6. Formulation of field problem

$$
\begin{gathered}
v=\frac{\delta \phi}{\delta t} \\
\phi=\int_{0}^{r} \mu H 2 \pi r d r \\
v=2 \pi \mu \int_{0}^{r} r \frac{\delta H}{\delta t} \\
J=\frac{\delta H}{\delta r} \\
J=\frac{v}{2 \pi r} \sigma
\end{gathered}
$$

The equation governing the relationship between the magnetic field and induced current is obtained by combining the above equations.

$$
\frac{\delta^{2} H}{\delta r^{2}}+\frac{1}{r} \frac{\delta H}{\delta r}-\mu \sigma \frac{\delta H}{\delta t}=0
$$

Under the condition of sinusoidal excitation with magnetic field

$$
H=H_{o} e^{-j\left(\omega t-\frac{2 \pi Z}{L}\right)}
$$

the governing equation takes the form of standard Bessel's equation

$$
\frac{\delta^{2} H}{\delta r^{2}}+\frac{1}{r} \frac{\delta H}{\delta r}-j \omega \mu \sigma H=0
$$

The solution to 15 is the standard Bessel equation of the following form

$$
H_{z}(r)=A H_{o}(\sqrt{j k r})
$$

where

$$
\begin{gathered}
k=\sqrt{\mu \sigma \omega} \\
H_{o}(\sqrt{j k r})=\operatorname{ber}(k r)+j b e i(k r) \\
\operatorname{ber}(k r)=1-\frac{(k r / 2)^{4}}{2 !}+\frac{(k r / 2)^{8}}{4 !}-\ldots \ldots \\
\operatorname{bei}(k r)=\frac{(k r / 2)^{2}}{(1 !)^{2}}-\frac{(k r / 2)^{6}}{(3 !)^{2}}+\frac{(k r / 2)^{10}}{(5 !)^{2}}-\ldots . . \\
H_{z}(r)=\frac{[b e r(k r)+j b e i(k r)]}{[b e r(k R)+j b e i(k R)]} H_{o} \sin \left(\omega t-\frac{2 \pi Z}{L}\right)
\end{gathered}
$$

which can be approximated to

$$
H_{z}(r)=H_{o} \sin \left(\omega t-\frac{2 \pi Z}{L}-\theta_{H}\right)
$$

If end effects are considered then,

$$
H_{z}(r)=C_{r} H_{o} \sin \left(\omega t-\frac{2 \pi Z}{L}-\theta_{H}\right)
$$

Thus it is clear that the axial field in the metal pool is lagging the ideal excitation field. Hence we arrive at an expression for the radial field which is proportional to the radius and leads the axial field by $90^{\circ}$ as

$$
H_{r}(r)=\frac{r}{R} H_{o} \sin \left(\omega t-\frac{2 \pi Z}{L}-\theta_{H}+90^{\circ}\right)
$$

Using 21 in 12 gives expression for $\mathbf{J}$ as,

$$
J_{\phi}(r)=C_{r} \frac{\left[(b e r)^{\prime}(k r)+j(b e i)^{\prime}(k r)\right]}{[(b e r)(k R)+j(b e i)(k R)]} H_{o} \sin \left(\omega t-\frac{2 \pi Z}{L}\right)
$$

which can be approximated to

$$
J_{\phi}(r)=C_{r} \frac{r}{R} J_{o} \sin \left(\omega t-\frac{2 \pi Z}{L}+\theta_{J}\right)
$$

$\theta_{H}$ and $\theta_{J}$ can be approximated to $0^{0}$ and $90^{\circ}$ respectively. The $\mathrm{J}$ field and $\mathrm{B}$ field in the metal pool interact to give radial 
and axial forces defined by the equation $F=J \times B$. The axial Power transferred to the metal pool is given by and radial forces are given by

$$
\begin{aligned}
F_{z} & =J_{\phi} B_{r} \\
& =\mu_{o} C_{r} J_{o} H_{o} \frac{r^{2}}{R^{2}} \sin \left(\omega t-\frac{2 \pi Z}{L}+90^{\circ}\right) \sin \left(\omega t-\frac{2 \pi Z}{L}+90^{\circ}\right) \\
& =\mu_{o} C_{r} J_{o} H_{o} \frac{r^{2}}{R^{2}} \frac{1-\cos 2[\omega t-(2 \pi Z) / L]}{2}
\end{aligned}
$$

$$
\begin{aligned}
F_{r}= & J_{\phi} B_{z} \\
& =\mu_{o} C_{r}^{2} J_{o} H_{o} \frac{r}{R} \sin \left(\omega t-\frac{2 \pi Z}{L}\right) \sin \left(\omega t-\frac{2 \pi Z}{L}-90^{\circ}\right) \\
& =\mu_{o} C_{r}{ }^{2} J_{o} H_{o} \frac{r}{R} \frac{\sin 2[\omega t-(2 \pi Z) / L]}{2}
\end{aligned}
$$

From 27 and 28 we can find that radial force is proportional to the radius and the axial force is proportional to the square of the radius. The radial force has no average whereas the axial force density averages in the direction of phase sequence.

\section{Power transferred to the metal pool}

As will be seen in the next section, the current density at low frequencies can be approximated to be varying linearly with the radius as shown in 7 . In such a situation it is possible to find the amount of heat transferred to the metal pool. If $J_{o}$ is
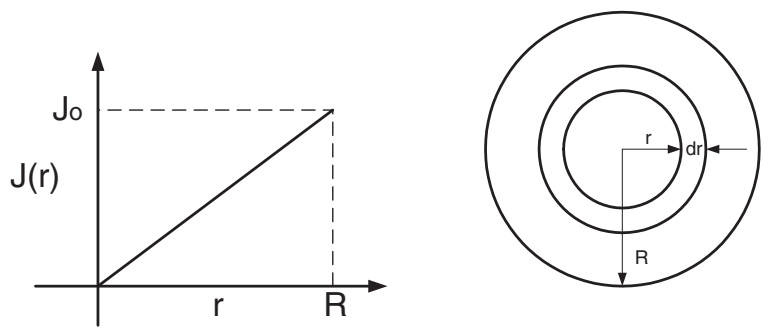

Fig. 7. Eddy current density v/s Radius for Al at low frequencies

the peak value of current density at radius $\mathrm{r}=\mathrm{R}$ (where $\mathrm{R}$ is the radius of Al billet), then at any radius $r<R$

$$
J=J_{o} \frac{r}{R}
$$

Consider an elemental cylinder of thickness $d r$ situated at a radius $r$. Power transferred to this elemental cylinder is given by

$$
\begin{aligned}
d W & =(J \cdot d A)^{2} \cdot d R \\
& =(J \cdot 2 \pi r d r)^{2} \cdot \frac{\rho L}{2 \pi r d r} \\
& =J^{2} \rho L 2 \pi r d r
\end{aligned}
$$

$$
\begin{aligned}
W & =\int_{0}^{R} d W \\
& =\int_{0}^{R} 2 \pi \rho L J^{2} r d r \\
& =\int_{0}^{R} \frac{2 \pi \rho L J_{0}{ }^{2}}{R^{2}} r^{3} d r \\
& =\frac{\pi \rho L J_{0}^{2} R^{4}}{2}
\end{aligned}
$$

\section{SAMPLE FIELD CALCULATIONS}

Table I lists the parameters used for obtaining field solutions through analytical and simulation model. Fig. 8 to 11 show

TABLE I

STIRRER PARAMETERS

\begin{tabular}{|c|c|}
\hline Coil Radius R & $0.042 \mathrm{~m}$ \\
\hline $\mathrm{L}$ & $0.16 \mathrm{~m}$ \\
\hline $\mathrm{N}$ & 10 \\
\hline Billet Diameter & $63.35 \mathrm{~mm}$ \\
\hline Frequency & $10,50 \mathrm{~Hz}$ \\
\hline Excitation Current & $200 \mathrm{~A}$ R.M.S \\
\hline$\mu_{r}$ of Aluminium & 1 \\
\hline$\sigma$ for aluminium & $3.8 \mathrm{e} 7 \mathrm{Sm}^{-1}$ \\
\hline
\end{tabular}

the variation of magnetic field intensity and eddy current density as a function of radius obtained analytically using Mathcad. It is observed that at lower frequency $(10 \mathrm{~Hz})$ the field is uniformly distributed along the radius whereas at higher frequency $(50 \mathrm{~Hz})$ due to skin effect the field intensities are concentrated at the periphery.

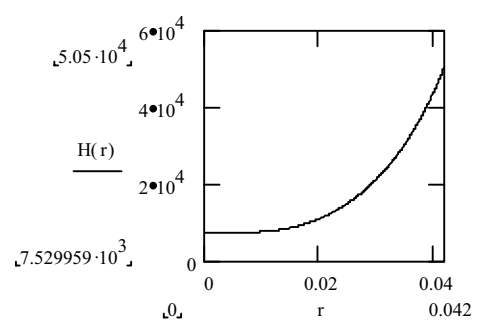

Fig. 8. Magnetic field Intensity(A/m) v/s Radius(m) at $50 \mathrm{~Hz}$

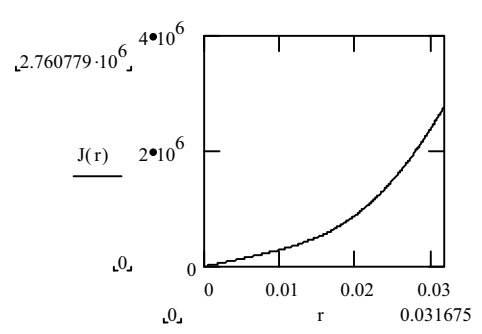

Fig. 9. Eddy Current Density $\left(\mathrm{A} / \mathrm{m}^{2}\right) \mathrm{v} / \mathrm{s} \operatorname{Radius}(\mathrm{m})$ in $\mathrm{Al}$ at $50 \mathrm{~Hz}$ 


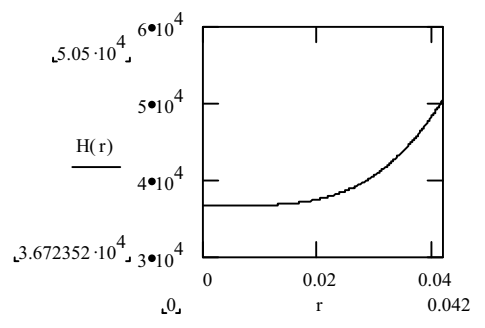

Fig. 10. Magnetic field Intensity (A/m) v/s Radius(m) for $\mathrm{Al}$ at $10 \mathrm{~Hz}$

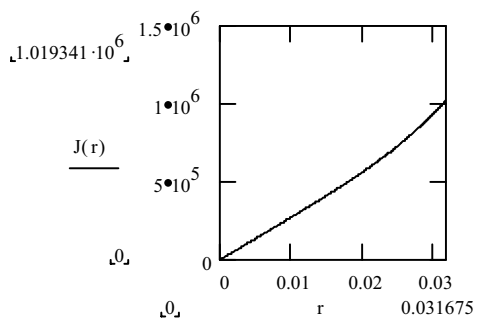

Fig. 11. Eddy Current Density $\left(\mathrm{A} / \mathrm{m}^{2}\right) \mathrm{v} / \mathrm{s} \operatorname{Radius}(\mathrm{m})$ in $\mathrm{Al}$ at $10 \mathrm{~Hz}$

\section{Analysis with Finite Element Model}

Fig. 12 shows the 3D mesh of the model generated by MagNet. The air box around the stirrer was omitted to show the parts of the stirrer viz. the coils and Aluminium mold. The tangential boundary condition $\partial \phi / \partial n=0$ is applied to the external surfaces of the air box where $\phi$ is the scalar potential and $n$ is the unit normal vector to the surface. The density of mesh was increased in the aluminium cylinder to improve the accuracy of solution. The geometry was solved using Time Harmonic 3D solver for billets of different sizes. Fig. 13 to 18 shows the field variations along the radial and axial directions for parameters listed in Table I.

The comparison between the magnetic field measured by analytical method and MagNet at $50 \mathrm{~Hz}$ are matching well but the current density is matching with an error of 26 percent . While at $10 \mathrm{~Hz}$, the magnetic field of aluminium is matching with an error of 36 percent but the current density is matching very well.

It was observed that the present arrangement was associated with large leakage flux. Hence teeth shaped structures of magnetic steel make were used to provide low reluctance path to the leakage flux. They additionally help in holding the coils

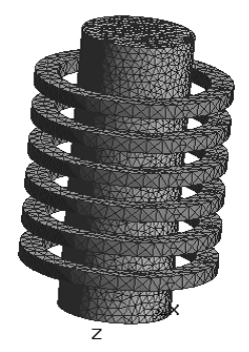

Fig. 12. 3D mesh of Finite element Model

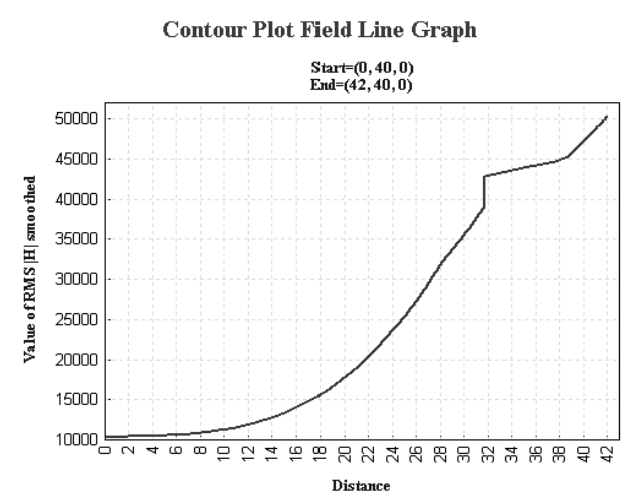

Fig. 13. Magnetic field Intensity(A/m) v/s Radius(mm) at $50 \mathrm{~Hz}$

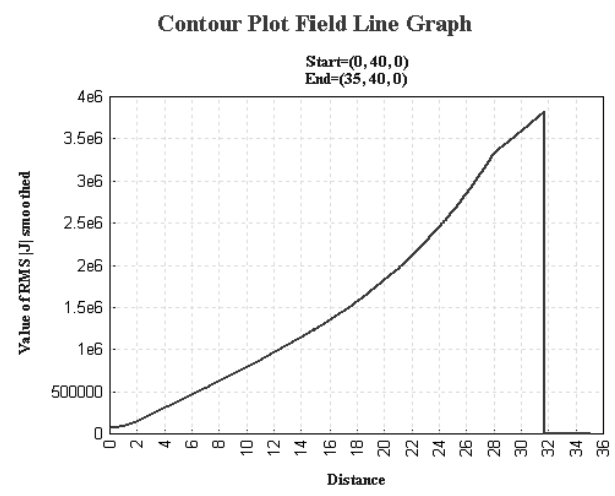

Fig. 14. Eddy Current density $\left(\mathrm{A} / \mathrm{m}^{2}\right) \mathrm{v} / \mathrm{s} \operatorname{Radius}(\mathrm{m})$ in $\mathrm{Al}$ at $50 \mathrm{~Hz}$

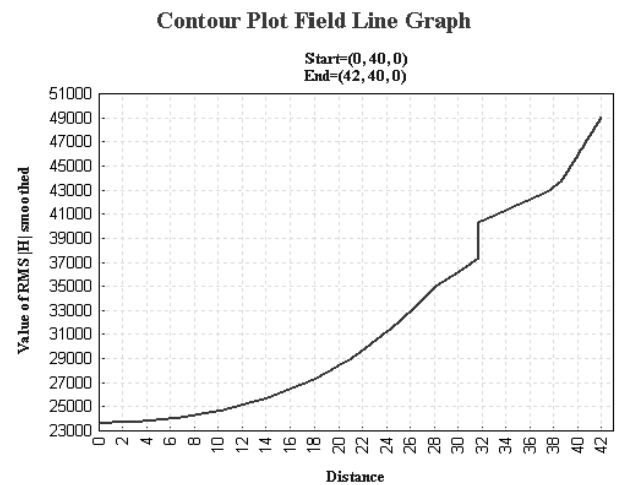

Fig. 15. Magnetic field Intensity(A/m) v/s Radius(mm) at 10Hz

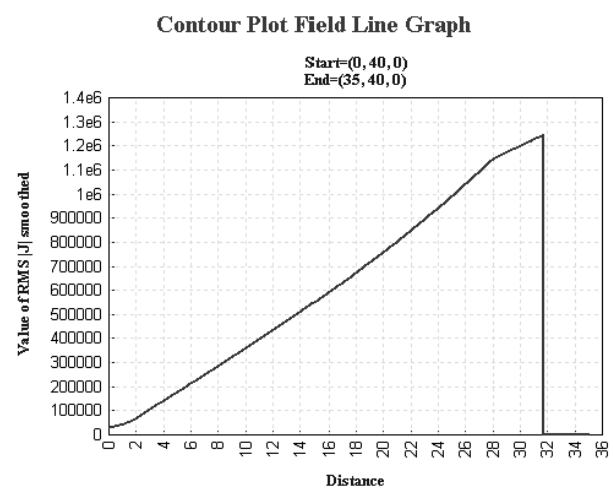

Fig. 16. Eddy Current density $\left(\mathrm{A} / \mathrm{m}^{2}\right) \mathrm{v} / \mathrm{s} \operatorname{Radius}(\mathrm{mm})$ in $\mathrm{Al}$ at $10 \mathrm{~Hz}$ 


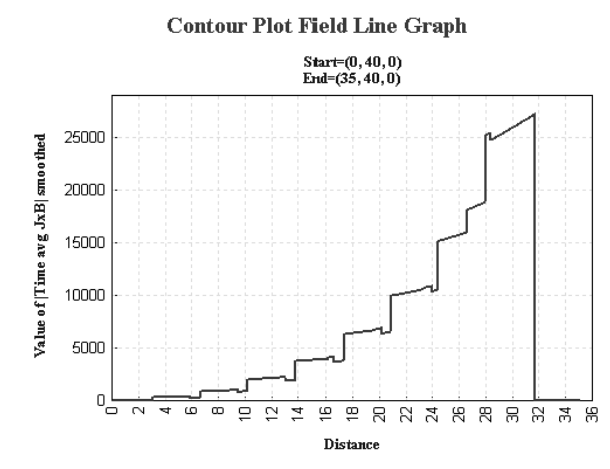

Fig. 17. Force density $\left(\mathrm{N} / \mathrm{m}^{3}\right) \mathrm{v} / \mathrm{s}$ radius $(\mathrm{mm})$ in $\mathrm{Al}$ at $10 \mathrm{~Hz}$

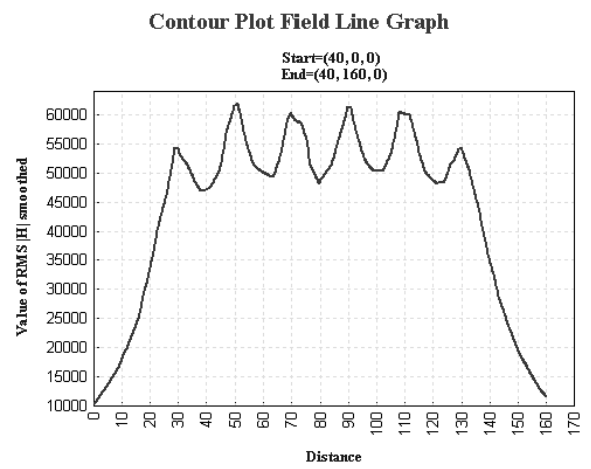

Fig. 18. Magnetic Field Intensity (A/m) along (Z)axis at $10 \mathrm{~Hz}$

in their position. Fig. 19 shows model geometry with six teeth shaped structures. Fig. 20 to 23 show the variation of field intensities along radial and axial directions with teeth shaped structures. It can be seen that the presence of teeth shaped structures significantly improved the magnitudes of $\mathrm{J}$ and B field resulting in higher volumetric force .

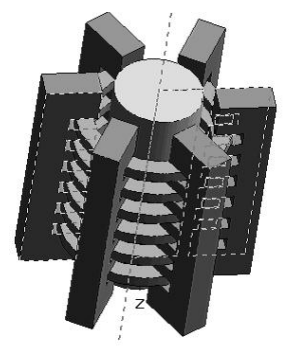

Fig. 19. Modified Finite element Model

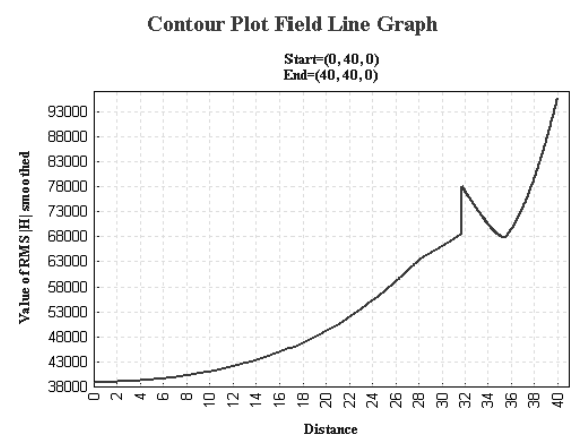

Fig. 20. Magnetic Field Intensity(A/m) v/s radius(mm) at $10 \mathrm{~Hz}$

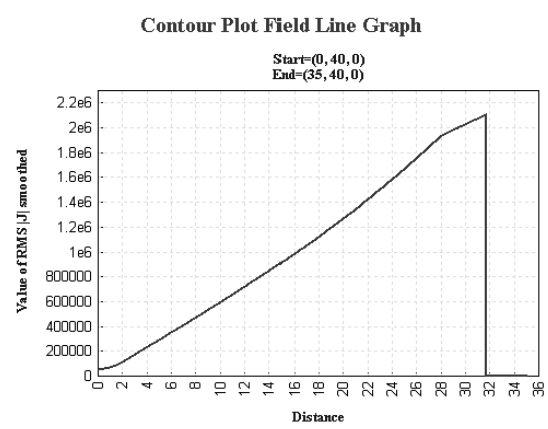

Fig. 21. Eddy Current density $\left(\mathrm{A} / \mathrm{m}^{2}\right) \mathrm{v} / \mathrm{s} \operatorname{radius}(\mathrm{mm})$ in $\mathrm{Al}$ at $10 \mathrm{~Hz}$

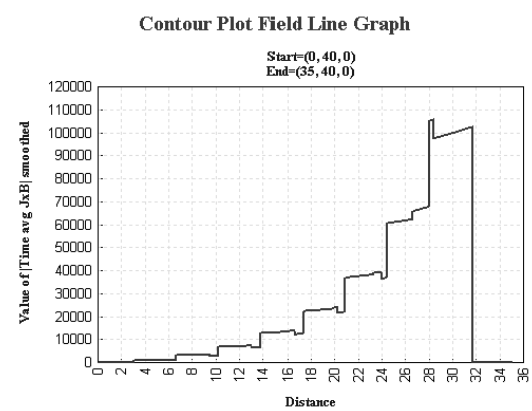

Fig. 22. Force density $\left(\mathrm{N} / \mathrm{m}^{3}\right) \mathrm{v} / \mathrm{s}$ radius $(\mathrm{mm})$ in $\mathrm{Al}$ at $10 \mathrm{~Hz}$

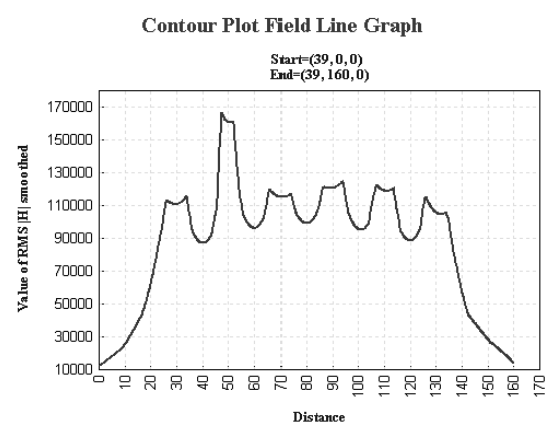

Fig. 23. Magnetic Field Intensity(A/m) along (Z)axis at $10 \mathrm{~Hz}$

\section{EXPERIMENTAL RESULTS}

Fig. 24 shows prototype of stirrer with $\mathrm{R}=0.042 \mathrm{~m}$ and $\mathrm{L}=0.16 \mathrm{~m}$ as its dimensions. Fig. 25 shows the block diagram

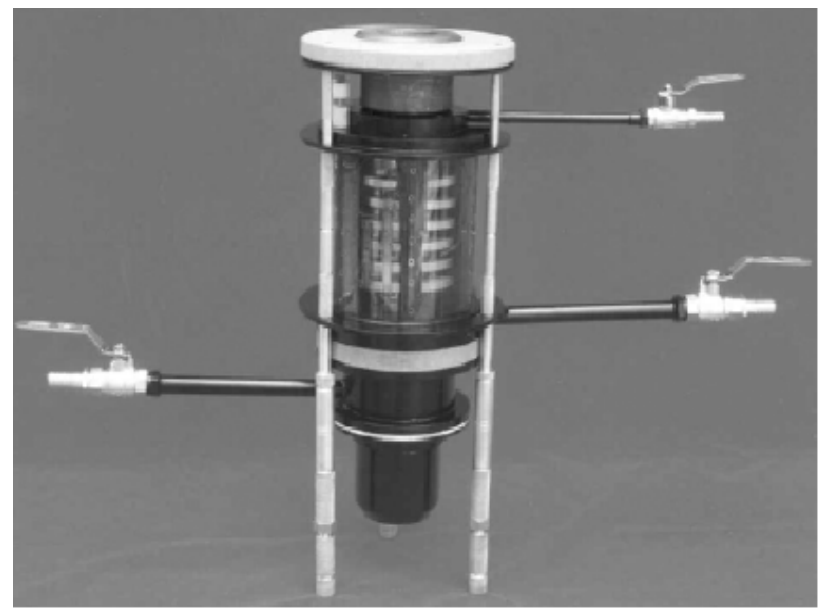

Fig. 24. Prototype of Stirrer 
of excitation system for the stirrer. Fig. 26 shows the variation of axial force with radius. Fig. 27 shows the variation of axial force density with excitation current for various billet diameters. As confirmed by analytical model it is nearly proportional to square of current. Fig. 28 shows the variation of axial force with frequency.

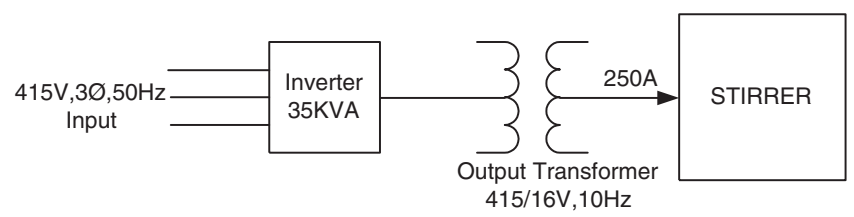

Fig. 25. Block Diagram of Excitation System for Stirrer

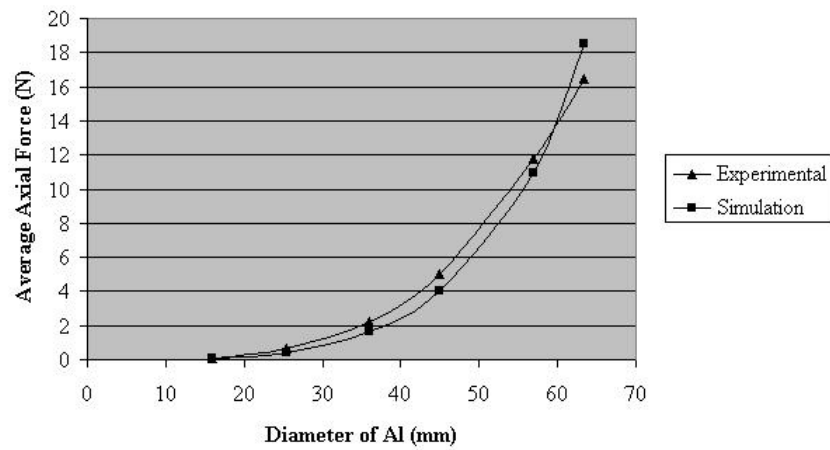

Fig. 26. Experimental Result of Force v/s Radius, I=200A, f=50Hz

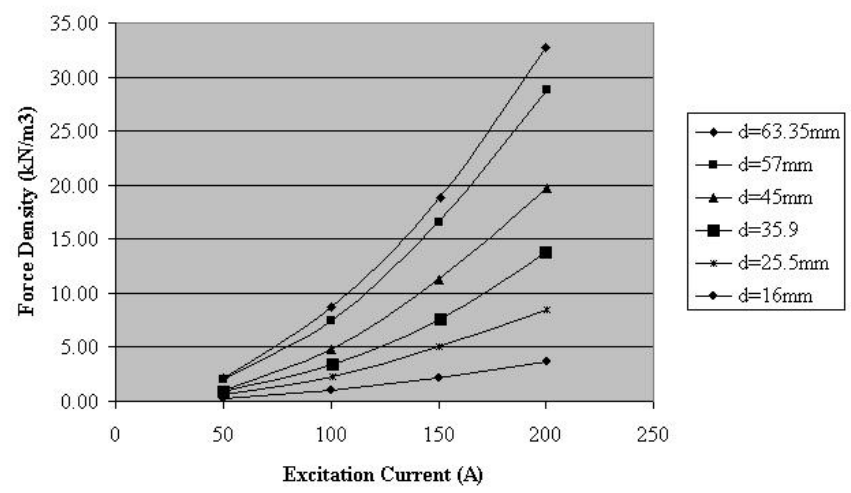

Fig. 27. Experimental Result of Force v/s Current, $D=63.35 \mathrm{~mm}, f=50 \mathrm{~Hz}$

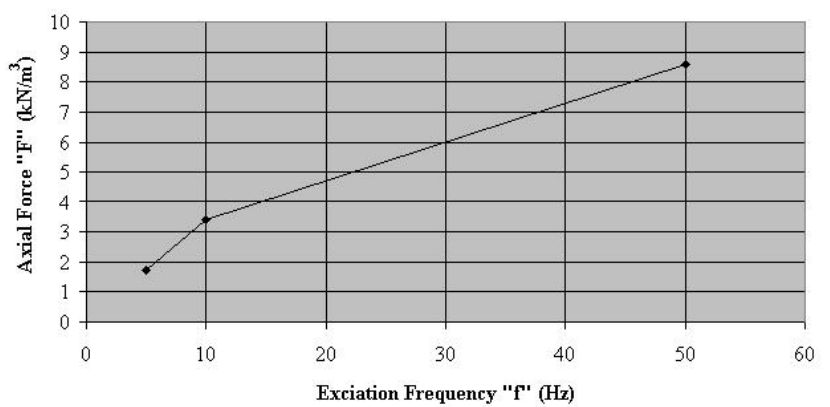

Fig. 28. Experimental Result of Force v/s Frequency, I=100A, D=63.35mm

\section{CONCLUSION}

This paper does not consider the MHD aspects of the molten metal. The field solutions obtained from analytical and simulation models are found to be matching quite well. We conclude that lower excitation frequencies give rise to uniform fields $(\mathrm{H})$ and linear currents $(\mathrm{J})$. Hence with this lower employed frequency will give gentle stirring of the charge (Molten metal) in the stirrer. Also at lower frequencies, the current density is distributed linearly as a function of the radius and the skin effect is barely noticeable. The uniform magnetic field and linear current density in the molten metal, will result in axial and radial forces. These axial and radial forces will be proportional to $\mathrm{r}$ and $r^{2}$ respectively with radial forces having no average while the axial forces averaging in the direction of phase sequence.

\section{ACKNOWLEDGMENT}

We acknowledge the help we received from Pramod Kumar (Ph.D student) of Mechanical Engineering Department during the course of this project. We are thankful to National Facility for Semi Solid Forming, Indian Institute of Science, for providing us with required facilities.

\section{REFERENCES}

[1] K. Fujisaki, J. Nakagawa and H. Misumi, "Fundamental Characteristics of Molten Metal Flow Control by Linear Induction Motor," IEEE Trans. Magn., vol.30, No.6, pp. 4764-4766, Nov. 1994.

[2] L. B. Trindade, A. C. F. Vilela, A. F. F. Filho, M. T. M. B. Vilhena and R. B. Soares, "Numerical Modelling of Electromagnetic Stirring for Continous Casting Billets," IEEE Trans. Magn., vol.38, No.6, pp. 36583660, Nov. 2002

[3] K. Fujisaki, T. Ueyama, T. Toh, M. Uehara and S. Kobayashi, " Magnetohydrodynamic calculation for electromagnetic stirring of molten metal," vol.34, No.4, pp. 2120-2122, July 1998 .

[4] J. Davies, " Induction heating handbook," McGraw Hill, Oct.1979.

[5] S. Yamamura, "Theory of Linear Induction Motors," University of Tokyo Press, 1978.

[6] A. G. Warren, "Mathematics Applied to electrical engineering," Chapman and Hall , 1942. 